\title{
A CANONICAL TRANSFORMATION NEAR A BOUNDARY POINT
}

\author{
L. SARASON ${ }^{1}$
}

ABSTRACT. A local homogeneous canonical transformation is constructed which straightens a curved boundary and freezes the coefficients of the principal part of a pseudo-differential operator in the neighborhood of a nonglancing ray.

Duistermaat and Hörmander [1] have studied the propogation along bicharacteristics of wave front sets of solutions of certain partial differential equations, using Fourier integral operators to effect a canonical transformation taking the given operator (locally) into $\partial / \partial x_{1}$. Hörmander [2] has also studied the problem with the aid of specially constructed pseudo-differential operators. Lax and Nirenberg [3] have applied the latter method to the study of boundary value problems, but thus far their approach has not handled the glancing ray case. As a first step towards adapting the approach of [1] to deal with boundary value problems, we construct a canonical transformation, away from glancing rays, which simultaneously reduces the boundary and the equation to a convenient form. I wish to thank Ralph Phillips for many helpful conversations.

Let $p(x, t ; \xi, \tau)$ be a real symbol which is positive homogeneous of degree $m \geq 0, m$ an integer, and with $(x, t, \xi, \tau) \in R^{n-1} \times R \times R^{n-1} \times R$. Let $0 \neq\left(\xi^{0}, \tau^{0}\right)$ satisfy $\partial p\left(0,0 ; \xi^{0}, \tau^{0}\right) / \partial \tau \neq 0$. Let $\Gamma$ be a smooth surface in $R^{n}$, passing through $(0,0)$, and such that the normal to $\Gamma$ at $(0,0)$ points in the direction of the $t$ axis.

Theorem. There is a canonical map $\chi:(x, t, \xi, \tau) \underline{\chi},(y, s, \eta, \sigma) \in R^{2 n}$, defined in a conic neighborbood $\mathcal{U}$ of $\left(0,0, \xi^{0}, \tau^{0}\right)$, bomogeneous of degree one in $(\xi, \tau)$, and such that for $(x, t, \xi, \tau) \leq \mathcal{U}$,

(i) $(x, t) \in \Gamma \Rightarrow s=0$,

(ii) $p(x, t ; \xi, \tau)=p(0,0 ; \eta, \sigma) \stackrel{\text { def }}{=} p_{0}(\eta, \sigma)$.

Received by the editors November 23, 1973.

AMS (MOS) subject classifications (1970). Primary 35 A30.

1 Sponsored by the United States Army under Contract No. DA-31-124-ARO-D- 462. 
Proof. For $\chi$ to be canonical means that the Poisson brackets of the image points satisfy

$$
\begin{gathered}
\left\{y_{i}, y_{j}\right\}=\left\{y_{i}, s\right\}=\left\{y_{i}, \sigma\right\}=\left\{s, \eta_{i}\right\}=\left\{\eta_{i}, \sigma\right\}=0, \\
\{s, \sigma\}=1, \quad\left\{y_{i}, \eta_{j}\right\}=\delta_{i j},
\end{gathered}
$$

where

$$
\begin{aligned}
\{u, v\} & =\sum_{l}\left(\frac{\partial u}{\partial x_{l}} \frac{\partial v}{\partial \xi_{l}}-\frac{\partial u}{\partial \xi_{l}} \frac{\partial v}{\partial x_{l}}\right)+\left(\frac{\partial u}{\partial t} \frac{\partial v}{\partial \tau}-\frac{\partial u}{\partial \tau} \frac{\partial v}{\partial t}\right) \\
& \stackrel{\text { def }}{=} H_{v} u \stackrel{\text { def }}{=}\left[b_{v}^{(1)} \cdot\left(\nabla_{x}, \frac{\partial}{\partial t}\right)-b_{v}^{(2)} \cdot\left(\nabla_{\xi}, \frac{\partial}{\partial \tau}\right)\right] u, \quad b_{v}=\left(b_{v}^{(1)}, b_{v}^{(2)}\right) .
\end{aligned}
$$

Our construction of $\chi$ is a modification of that given by Duistermaat and Hörmander [1] in free space. The functions $\eta_{i}$ will be constructed successively on $\Gamma \times R^{n}$ by assigning each on an initial manifold transverse to the linear span of those $b_{\eta}$ which are already known, and such that $h_{\eta_{i}}^{(1)}$ is tangential to $\Gamma$. This last fact will enable us to construct $s$ such that $s=0$ on $\Gamma$. Once $\eta$ is constructed, $\sigma$ is determined by (ii). To extend $\eta$ and $\sigma$ off of $\Gamma$, we shall use the equation $b_{p} \eta=0$ together with (ii); a simple application of the chain rule shows that this construction implies the canonical relations $\left\{\eta_{i}, \sigma\right\}=0$. Finally, we shall use the initial condition $(y, s)(0,0, \eta, \sigma)=(y, s)$ together with the equations $b_{\eta_{i}}(y, s)=$ $b_{\sigma}(y, s)=0$ to determine $(y, s)$.

We now construct $\chi$. Let $N_{1}$ be a neighborhood of $(0,0)$ in $\Gamma$, and $C_{1}$ a conic neighborhood of $\left(\xi^{0}, \tau^{0}\right)$ in $R^{n}$ such that for $(x, t) \in N_{1}$ and $0 \neq(\xi, \tau) \in C_{1}$,

$$
\left\langle n, \nabla_{\xi, \tau}\right\rangle p \neq 0,
$$

where $n$ is the normal to $\Gamma$ at $(x, t)$. On $N_{1}$, let $v_{1}(x, t)$ be a nonsingular tangential vector field such that $\left\langle\left(\xi^{0}, \tau^{0}\right), v_{1}(0,0)\right\rangle=\xi_{1}^{0}$, and define

$$
\eta_{1}(x, t, \xi, \tau)=\left\langle(\xi, \tau), v_{1}(x, t)\right\rangle, \quad(x, t, \xi, \tau) \in N_{1} \times C_{1} .
$$

Because of (1), $b_{p}^{(1)}$ is not tangential to $N_{1}$, and hence we can extend the definition of $\eta_{1}$ off of $N_{1} \times C_{1}$ by using (2) as an initial condition for

$$
\left\{\eta_{1}, p\right\} \equiv H_{p} \eta_{1}=0
$$

We define recursively triples $\left\{N_{i}, v_{i}, \eta_{i}\right\}, i=2, \cdots, n-1$ as follows. 
Let $N_{i} \ni(0,0)$ be a smooth $(n-1)$-dimensional surface in $N_{i-1}$, transverse to the span of the vectors $v_{1}, \cdots, v_{i-1}$ and not orthogonal to $\left(\xi^{0}, \tau^{0}\right)$ unless $\xi_{i}^{0}=\xi_{i+1}^{0}=\cdots=\xi_{n-1}^{0}=0$. Let $v_{i}$ be a nonsingular vector field in $N_{i}$ such that $\left\langle\left(\xi^{0}, \tau^{0}\right), v_{i}(0,0)\right\rangle=\xi_{i}^{0}$. Define

$$
\eta_{i}=\left\langle(\xi, \tau), v_{i}(x, t)\right\rangle, \quad(x, t, \xi, \tau) \in N_{i} \times C_{1},
$$

and extend $\eta_{i}$ by the equations.

$$
\left\{\eta_{i}, \eta_{j}\right\}=0, \quad j<i
$$

and

$$
\left\{\eta_{i}, p\right\}=0
$$

The consistency of the construction of $\eta$ using (5) and (6) follows from the identity $\left[H_{u}, H_{v}\right]=H_{\{u, v\}}$. For example, the equations $\left\{\eta_{j}, \eta_{k}\right\}=0$ are satisfied by construction along a submanifold $M_{k}, j<k$, and $\left\{\eta_{j}, p\right\}=$ $\left\{\eta_{k}, p\right\}=0$ along integral curves of $H_{p}$ through $M_{k}$. On these integral curves, then,

$$
\left.\left\{p,\left\{\eta_{j}, \eta_{k}\right\}\right\}=H_{\left\{\eta_{j}, \eta_{k}\right.}\right\} p=\left[H_{\eta_{j}}, H_{\eta_{k}}\right] p=H_{\eta_{j}}\left\{p, \eta_{k}\right\}-H_{\eta_{k}}\left\{p, \eta_{j}\right\}=0,
$$

so that $\left\{\eta_{j}, \eta_{k}\right\}=0$ along these curves.

Remark. If $\Gamma$ is the hyperplane $t=0$, it suffices to set $\eta=\xi$ on $N_{1} \times C_{1}$ and use (6) to extend the definition of $\eta_{i}$

The condition $\partial p\left(0,0 ; \xi^{0}, \tau^{0}\right) / \partial \tau \neq 0$, together with the above construction, ensures that there is a conic neighborhood $\mathcal{U}$ of $\left(0,0 ; \xi^{0}, r^{0}\right)$ in which $\sigma$ is uniquely defined by (ii) if we set $\sigma\left(0,0 ; \xi^{0}, \tau^{0}\right)=\tau^{0}$. Locally, $\sigma$ is defined as a function of $Z \equiv(\eta, p)$, from which we conclude that

$$
\left\{\sigma, \eta_{j}\right\}=H_{\eta_{j}} \sigma=\sum_{k} \frac{\partial \sigma}{\partial \eta_{k}}\left\{\eta_{j}, \eta_{k}\right\}+\frac{\partial \sigma}{\partial p}\left\{\eta_{j}, p\right\}=0 .
$$

According to [1], we can now determine $(y, s)$ in $\mathcal{U}$ by assigning $(y, s)$ on an $n$-dimensional manifold transverse to the span of $b_{\eta_{j}}, j=1, \cdots, n-1$, and $b_{\sigma}$, provided that these vectors together with the radial vector $(0,0 ; \xi, \tau)$ are linearly independent. Such a manifold is the subspace $x=0, t=0$. To see this, we need only observe that

$$
b_{\eta_{j}}(0,0, \xi, \tau)=\left(e_{j}, 0,0, \partial \eta_{j} / \partial t\right)
$$

where $e_{j}$ is a standard unit basis vector in $R^{n-1}$, and that the $n$th component of $h_{\tau}(0,0, \xi, \tau)$ is (cf. (ii)) 


$$
\frac{\partial \sigma}{\partial \tau}(0,0, \xi, \tau)=\frac{\partial p_{0}}{\partial \sigma}\left\{\frac{\partial p}{\partial \tau}-\sum \frac{\partial p_{0}}{\partial \eta_{j}} \frac{\partial \eta_{j}}{\partial \tau}\right\}=\frac{\partial p_{0}}{\partial \sigma} \frac{\partial p}{\partial \tau}(0,0, \xi, \tau) \neq 0 .
$$

We as sign initial conditions

$$
(y, s)(0,0, \xi, \tau)=(0,0) .
$$

Using (7) together with equations $H_{\eta_{i}}(y, s)=\left(e_{i} 0\right), H_{\sigma}(y, s)=(0,1)$, serves to define $(y, s)$ in $U$.

There remains to show that (i) holds. But $s$ is invariant on the integral curves of each $H_{\eta_{j}}$, and if $(x, t) \in N_{j}$, then $h_{\eta_{j}}^{(1)}=v_{j}$ is tangent to $\Gamma$. Given $\left(x^{\prime}, t^{\prime}, \xi^{\prime}, \tau^{\prime}\right) \in \mathcal{U}$ with $\left(x^{\prime}, t^{\prime}\right) \in N_{1}$, we follow successively the integral curves of $H_{\eta_{i}}, i=1, \cdots, n-1$, through $\left(x^{i}, t^{i}, \xi^{i}, \tau^{i}\right)$ till $(x, t)$ hits $N_{i+1}$ at $\left(x^{i+1}, t^{i+1}\right)$ and $(\xi, \tau)=\left(\xi^{i+1}, \tau^{i+1}\right)$, with $N_{n}$ defined as the point $(0,0)$. We conclude that for some $P=\left(0,0, \xi^{n}, \tau^{n}\right) \in \mathcal{U}, s(x, t, \xi, \tau)=$ $s(P)=0$ by (7). Theorem 1 is proved.

As a corollary of the proof, we note that if $\Gamma$ is the hyperplane $t=0$, then $X$ can be extended to a conical neighborhood of any cone $C=$ $(0,0, V \backslash\{0\})$, where $V$ is a closed simply connected cone, and where $\partial p / \partial \tau \neq 0$ on $C \backslash\{0\}$. Since $\chi(0,0, \xi, \tau)=(0,0, \xi, \tau)$, condition (ii), together with a simple homotopy argument, allows us to drop the as sumption that $V$ be simply connected.

Remark. If $p$ has the parity of $m$, then $\chi$ extends by homogeneity to a two-sided conic set.

Some applications and extensions will be reported on elsewhere.

\section{REF ERENCES}

1. J. J. Dui stermaat and L. Hörmander, Fourier integral operators. II, Acta Math. 128 (1972), 183-269.

2. L. Hörmander, On the existence and regularity of solutions of linear pseudodifferential equations, Enseignement Math. 17 (1971), 99-163.

3. L. Nirenberg, Lectures on linear partial differential equations, Conference Board of the Mathematical Sciences, Regional Conference Ser. in Math., vol. 17 (to appear).

DEPA RTMENT OF MA THEMATICS, UNIVE RSITY OF WASHINGTON, SEATTLE, WASHINGTON 98105 